Then

$$
U_{1}=\left[\begin{array}{l}
1 \\
4 \\
5
\end{array}\right], U_{2}=\left[\begin{array}{l}
1 \\
4 \\
7
\end{array}\right] \text {, and } U_{3}=\left[\begin{array}{l}
3 \\
6 \\
4
\end{array}\right]
$$

Thus

$$
\begin{aligned}
& B=\left[\begin{array}{lll}
1 & 0 & 0 \\
0 & 1 & 0 \\
0 & 0 & 1
\end{array}\right]+\left[\begin{array}{l}
1 \\
4 \\
5
\end{array}\right]\left[\begin{array}{lll}
1 & 0 & 0
\end{array}\right]+\left[\begin{array}{l}
1 \\
4 \\
7
\end{array}\right]\left[\begin{array}{lll}
0 & 1 & 0
\end{array}\right]+\left[\begin{array}{l}
3 \\
6 \\
4
\end{array}\right]\left[\begin{array}{lll}
0 & 0 & 1
\end{array}\right] . \\
& C_{0}=I, C_{0}^{-1}=I \text {. } \\
& C_{1}^{-1}=I-\left(I U_{1}\right)\left(V_{1}{ }^{T} I\right) /\left(1+V_{1}{ }^{T} I U_{1}\right)=I-\frac{1}{2}\left[\begin{array}{lll}
1 & 0 & 0 \\
4 & 0 & 0 \\
5 & 0 & 0
\end{array}\right]=\left[\begin{array}{ccc}
\frac{1}{2} & 0 & 0 \\
-2 & 1 & 0 \\
-\frac{5}{2} & 0 & 1
\end{array}\right] \text {. } \\
& C_{2}^{-1}=C_{1}^{-1}-\frac{\left(\left[\begin{array}{ccc}
\frac{1}{2} & 0 & 0 \\
-2 & 1 & 0 \\
-\frac{5}{2} & 0 & 1
\end{array}\right]\left[\begin{array}{l}
1 \\
4 \\
7
\end{array}\right]\right)\left(\left[\begin{array}{ccc}
0 & 1 & 0
\end{array}\right]\left[\begin{array}{ccc}
\frac{1}{2} & 0 & 0 \\
-2 & 1 & 0 \\
-\frac{5}{2} & 0 & 1
\end{array}\right]\right)}{1+\left[\begin{array}{lll}
0 & 1 & 0
\end{array}\right]\left(\left[\begin{array}{ccc}
\frac{1}{2} & 0 & 0 \\
-2 & 1 & 0 \\
-\frac{5}{2} & 0 & 1
\end{array}\right]\left[\begin{array}{l}
1 \\
4 \\
7
\end{array}\right]\right)} . \\
& C_{3}^{-1}=C_{2}^{-1}-\left(C_{2}^{-1} U_{3}\right)\left(V_{3}{ }^{T} C_{2}^{-1}\right) /\left(1+V_{3}{ }^{T} C_{2}^{-1} U_{3}\right)=B^{-1} \text {. }
\end{aligned}
$$

U. S. Navy Electronics Laboratory

San Diego, California

1. A. Ralston \& H. S. Wilf, Mathematical Methods for Digital Computers, Wiley, New York, 1960, pp. 73-77. MR 22 *8680.

2. A. S. Housenolder, Principles of Numerical Analysis, McGraw-Hill, New York, 1953, pp. 79, 83. MR 15, 470 .

\title{
Certain Expansions of the Basic Hypergeometric Functions
}

\section{By Arun Verma}

1. Introduction. In a recent paper Jerry L. Fields and Jet Wimp [7] have used a very elegant method by induction through Laplace transform to derive a number of expansions of hypergeometric functions. In this paper, I have used certain basic integrals and the method of induction to derive certain expansions of basic hypergeometric functions of a very general character. The following usual notation has been used throughout the paper. Let

$$
[a]_{n}=\left[q^{a}\right]_{n}=\left(1-q^{a}\right)\left(1-q^{a+1}\right) \cdots\left(1-q^{a+n-1}\right), \quad[a]_{0}=1,
$$

and

$$
(a+x)_{\lambda}=\prod_{k=0}^{\infty}\left(\frac{a+x q^{k}}{a+x q^{k+\lambda}}\right), \quad(1-x)_{\infty}=\prod_{n=0}^{\infty}\left(1-x q^{n}\right) .
$$

Received May 24, 1965. 
Also, let

$$
{ }_{r} \Phi_{s}\left[\begin{array}{c}
\left(a_{r}\right) ; x \\
\left(b_{s}\right)
\end{array}\right]=\sum_{n=0}^{\infty} \frac{\left[\left(a_{r}\right)\right]_{n} x^{n}}{[1]_{n}\left[\left(b_{s}\right)\right]_{n}}, \quad|q|<1 \text { and }|x|<1
$$

and

$$
B_{q}(\alpha, \beta)=\mathrm{S}_{0}{ }^{1} t^{\alpha-1}(1-q t)_{\beta-1} d(q, t)
$$

where

$$
\frac{1}{1-q} \mathbf{S}_{0}^{x} f(y) d(q, y)=x \sum_{i=0}^{\infty} q^{i} f\left(q^{i} x\right)
$$

and $\left(a_{r}\right)$ means $r$ parameters of the type $a_{1}, a_{2}, \cdots, a_{r}$.

The basic analogue of the Laplace transform $[4,5]$ is defined as

$$
\begin{aligned}
g(s)={ }_{q} L_{8} f(x)= & \frac{1}{1-q} \mathrm{~S}_{0}{ }^{-1} E_{q}(q s x) f(x) d(q, x) \\
& =\frac{(1-q)}{s} \sum_{i=0}^{\infty} q^{i} f\left(s^{-1} q^{i}\right) /(1-q)_{i} .
\end{aligned}
$$

The inverse is defined by the complex integral

$$
f(x)=\frac{1}{2 \pi i} \int_{c} g(s) e_{q}(s x) d s,
$$

where the path of integration $C$ encircles the origin, and can also be deformed into a loop, parallel to the imaginary axis. Also

$$
\begin{aligned}
& E_{q}(x)=\sum_{r=0}^{\infty} \frac{(-x)^{r} q^{(r-1) / 2 r}}{[1]_{r}}, \\
& e_{q}(x)=\sum_{r=0}^{\infty} \frac{x^{r}}{[1]_{r}}
\end{aligned}
$$

2. We now proceed to derive the following expansion

$$
\begin{aligned}
& { }_{p+r} \Phi_{l+m}\left[\begin{array}{c}
\left(a_{p}\right),\left(c_{r}\right) ; x w \\
\left(b_{1}\right),\left(d_{m}\right)
\end{array}\right]=\sum_{n=0}^{\infty} \frac{\left[\left(a_{p}\right)\right]_{n}[\alpha]_{n}[\beta]_{n}(-x)^{n} q^{n(n-1) / 2}}{[1]_{n}\left[\left(b_{l}\right)\right]_{n}[\gamma+n]_{n}} \\
& \times{ }_{p+2} \Phi_{l+1}\left[\begin{array}{c}
n+\alpha, n+\beta, n+\left(a_{p}\right) ; x \\
2 n+\gamma+1, n+\left(b_{l}\right)
\end{array}\right] \times{ }_{r+2} \Phi_{m+2}\left[\begin{array}{c}
-n, n+\gamma,\left(c_{r}\right) ; w q \\
\alpha, \beta,\left(d_{m}\right)
\end{array}\right] .
\end{aligned}
$$

To prove this result, we first prove that

$$
\begin{aligned}
{ }_{2} \Phi_{1}\left[\begin{array}{c}
A, B ; x w / q \\
C
\end{array}\right] & =\sum_{m=0}^{\infty} \frac{[a]_{m}[b]_{m} q^{m(m-1) / 2}(-x)^{m}}{[1]_{m}[\gamma+m-1]_{m}} \\
\times{ }_{2} \Phi_{1} & {\left[\begin{array}{c}
a+m, b+m ; x \\
\gamma+2 m
\end{array}\right] \cdot{ }_{4} \Phi_{3}\left[\begin{array}{c}
A, B, \gamma+m-1,-m ; w \\
C, a, b
\end{array}\right] . }
\end{aligned}
$$

Proof of (2.2). The right-hand side of (2.2) equals to

$$
\sum_{m=0}^{\infty} \sum_{n=0}^{\infty} \frac{(-)^{m} x^{m+n} q^{m(m-1) / 2}[a]_{m+n}[b]_{m+n}}{[1]_{m}[1]_{n}[\gamma+m-1]_{m}[\gamma+2 m]_{n}} \cdot{ }_{4} \Phi_{3}\left[\begin{array}{c}
A, B, \gamma+m-1,-m ; w \\
C, a, b
\end{array}\right]
$$


Setting $m+n=r$, and changing the order of summation, we get

$$
\sum_{r=0}^{\infty} \frac{[a]_{r}[b]_{r}}{[1]_{r}} x^{r} \sum_{m=0}^{r}[-r]_{m} q^{m r}{ }_{4} \Phi_{3}\left[\begin{array}{c}
A, B, \gamma+m-1,-m ; w \\
C, a, b
\end{array}\right]
$$

The inner series equals to

$$
\begin{aligned}
\sum_{m=0}^{r} & \frac{[-r]_{m} q^{m r}}{[1]_{m}[\gamma+m-1]_{m}[\gamma+2 m]_{r-m}} \sum_{t=0}^{m} \frac{[A]_{t}[B]_{t}[\gamma+m-1]_{t}[-m]_{t} w^{t}}{[1]_{t}[C]_{t}[a]_{t}[b]_{t}}, \\
& =\sum_{s=0}^{r} \frac{[A]_{\ell}[B]_{\ell}[\gamma-1]_{s} w^{s}}{[1]_{s}[C]_{s}[a]_{s}[b]_{s}[\gamma]_{r}} \sum_{m=s}^{r} \frac{[-r]_{m}[\gamma-1+s]_{m}[-m]_{\delta}\left[\frac{1}{2} \gamma+\frac{1}{2}\right]_{m}\left[\frac{1}{2} \gamma+\frac{1}{2}\right]_{m}^{*} q^{m r}}{[1]_{m}[\gamma+r]_{m}\left[\frac{1}{2} \gamma-\frac{1}{2}\right]_{m}\left[\frac{1}{2} \gamma-\frac{1}{2}\right]_{m}^{*}},
\end{aligned}
$$

where $[a]_{m}^{*}=\left(1+q^{a}\right)\left(1+q^{a+1}\right)\left(1+q^{a+2}\right) \cdots\left(1+q^{a+m-1}\right)$.

Now setting $m=s+l$, we get

$$
\begin{aligned}
=\frac{1}{[\gamma]_{r}} \sum_{s=0}^{r} \frac{[A]_{s}[B]_{s}[-1]_{s}[-r]_{s}\left[\frac{1}{2} \gamma+\frac{1}{2}\right]_{s}\left[\frac{1}{2} \gamma+\frac{1}{2}\right]_{s}^{*}[\gamma-1+s]_{s}(-w)^{s}}{[1]_{\theta}[C]_{s}[a]_{s}[b]_{s}[\gamma+r]_{s}\left[\frac{1}{2} \gamma-\frac{1}{2}\right]_{s}\left[\frac{1}{2} \gamma-\frac{1}{2}\right]_{s}^{*}} \\
\quad \times q^{r s-k(s+1) / 2}{ }_{4} \Phi_{3}\left[\begin{array}{c}
\gamma-1+2 s,\left[\frac{1}{2} \gamma+\frac{1}{2}+s\right],\left[\frac{1}{2} \gamma+\frac{1}{2}+s\right]^{*},-r+s ; q^{r-s} \\
{\left[\frac{1}{2} \gamma-\frac{1}{2}+s\right],\left[\frac{1}{2} \gamma-\frac{1}{2}+s\right]^{*}, \gamma+r+s}
\end{array}\right] .
\end{aligned}
$$

But by a particular case of the sum of the well-poised ${ }_{6} \Phi_{5}$ it can be easily seen that the above ${ }_{4} \Phi_{3}$ vanishes except for $r=s$, when it reduces to unity. Hence the above expression, after a little simplification becomes

$$
\frac{[A]_{r}[B]_{r} q^{-r} w^{r}}{[C]_{r}[a]_{r}[b]_{r}}
$$

Substituting this value of the inner series in (2.3), we get the result. For $w=q$ this result reduces to a result due to Agarwal $[3 ; 3.2]$. Further letting $A, B, C$ tend to infinity, we get the basic analogue of a result due to Luke and Coleman $[9 ; 1.8]$.

Now we proceed to prove (2.1) by induction. Suppose (2.1) holds for some values of $p, r, l$ and $m$. Multiplying both sides by $x^{\sigma-1}$, and taking the $q$-analogue of Laplace transform (1.1) on both the sides and making use of the result [5; 9.2]:

$$
\frac{1}{1-q} \mathrm{~S}_{0}^{s-1} E_{q}(q s x) x^{k-1} d(q, x)=s^{-k} \prod_{j=0}^{\infty}\left(\frac{1-q^{j+1}}{1-q^{j+k}}\right), \quad[\mathrm{Rl}(k)>0] .
$$

We get that

$$
\begin{aligned}
{ }_{p+r+1} \Phi_{l+m}\left[\begin{array}{c}
\left.\left(a_{p}\right),\left(c_{r}\right), \sigma ; w / s\right] \\
\left(b_{l}\right),\left(d_{m}\right)
\end{array}\right]=\sum_{n=0}^{\infty} & \frac{\left[\left(a_{p}\right)\right]_{n}[\alpha]_{n}[\beta]_{n}[\sigma]_{n}(-s)^{-n}}{[1]_{n}[\gamma+n]_{n}\left[\left(b_{l}\right)\right]_{n}} \\
& \times q^{n(n-1) / 2}{ }_{r+2} \Phi_{m+2}\left[\begin{array}{c}
-n, n+\gamma,\left(c_{r}\right) ; w q \\
\alpha, \beta,\left(d_{m}\right)
\end{array}\right] \\
& \cdot{ }_{p+3} \Phi_{l+1}\left[\begin{array}{c}
\left.n+\alpha, n+\beta, n+\left(a_{p}\right), n+\sigma ; 1 / s\right], \\
2 n+\gamma+1, n+\left(b_{l}\right)
\end{array}\right],
\end{aligned}
$$

for $\operatorname{Rl}(\sigma)>0$. Thus replacing $s$ by $(1 / x)$ the induction with respect to ' $p$ ' is seen to be true. To effect the induction with respect to ' $l$ ' replace $x$ by $(1 / x)$ in $(2.1)$ and 
then multiplying both sides by $x^{-\sigma}$ and taking the inverse of the $q$-analogue of the Laplace transform (1.2), we get

$$
\begin{aligned}
&{ }_{p+r} \Phi_{l+m+1} {\left[\begin{array}{c}
\left(a_{p}\right),\left(c_{r}\right) ; w s \\
\left(b_{l}\right),\left(d_{m}\right), \sigma
\end{array}\right]=\sum_{n=0}^{\infty} \frac{\left[\left(a_{p}\right)\right]_{n}[\alpha]_{n}[\beta]_{n}(-s)^{n} q^{n(n-1) / 2}}{[1]_{n}\left[\left(b_{l}\right)\right]_{n}[\gamma+n]_{n}[\sigma]_{n}} } \\
& \times{ }_{{ }_{+}+2} \Phi_{m+2}\left[\begin{array}{c}
-n, n+\gamma,\left(c_{r}\right) ; w q \\
\alpha, \beta,\left(d_{m}\right)
\end{array}\right] \cdot{ }_{p+2} \Phi_{l+2}\left[\begin{array}{c}
n+\alpha, n+\beta, n+\left(a_{p}\right) ; s \\
2 n+\gamma+1, n+\left(b_{l}\right), n+\sigma
\end{array}\right] .
\end{aligned}
$$

Similarly, the induction with respect to $r, m$ can be effected. But for $p=l=0$, $r=2, m=1$ and $c_{1}=A, c_{2}=B, d_{1}=C$ and $w=w / q$ in (2.1) we obtain (2.2), and hence the proof by induction of (2.1) is complete. The conditions on the parameters can be waved off by analytic continuation.

(2.1) is the $q$-analogue of a result due to Fields and Wimp [7;1.3]. If we let $\alpha$ and $\beta$ tend to infinity and take $r=0, m=1$, we get the basic analogue of a result due to Toscano $[8 ; 1]$.

3. In this section, I proceed to derive, with the help of (2.1), another expansion of a more general character. Putting $w=0$, replacing $p$ by $(p-2)$ and setting $\alpha=a_{p-1}, \beta=a_{p}$ in (2.1), we get

$$
1=\sum_{n=0}^{\infty} \frac{\left[\left(a_{p}\right)\right]_{n}(-x)^{n} q^{n(n-1) / 2}}{[1]_{n}\left[\left(b_{l}\right)\right]_{n}[\gamma+n]_{n}}{ }_{p} \Phi_{l+1}\left[\begin{array}{c}
n+\left(a_{p}\right) ; x \\
2 n+\gamma+1, n+\left(b_{l}\right)
\end{array}\right] .
$$

Replacing $\left(a_{p}\right),\left(b_{l}\right), \gamma$ by $\left(a_{p}\right)+k,\left(b_{l}\right)+k$ and $\gamma+2 k$ respectively and multiplying both sides by $\left[\left(c_{r}\right)\right]_{k}(w x)^{k} /[1]_{k}\left[\left(d_{m}\right)\right]_{k}$, then setting $n+k=t$, we get

$$
\begin{aligned}
\frac{\left[\left(c_{r}\right)\right](w x)^{k}}{[1]_{k}\left[\left(d_{m}\right)\right]_{k}}=\frac{\left[\left(b_{l}\right)\right]_{k}\left[\left(c_{r}\right)\right]_{k}(q w)^{k}}{[1]_{k}\left[\left(a_{p}\right)\right]_{k}\left[\left(d_{m}\right)\right]_{k}} \sum_{t=k}^{\infty} & \frac{\left[\left(a_{p}\right)\right]_{t}[-t]_{k}[\gamma+t]_{k}}{[1]_{t}\left(\left(b_{l}\right)\right]_{t}[\gamma+t]_{t}} \\
& \times(-x)^{t} q^{t(t-1) / 2}{ }_{p} \Phi_{l+1}\left[\begin{array}{c}
t+\left(a_{p}\right) ; x \\
2 t+\gamma+1,\left(b_{l}\right)+t
\end{array}\right] .
\end{aligned}
$$

Summing both sides from $k=0$ to $\infty$, and changing the order of summation on the right-hand side, we have

$$
\begin{aligned}
&{ }_{r} \Phi_{m}\left[\begin{array}{c}
\left(c_{r}\right) ; w x \\
\left(d_{m}\right)
\end{array}\right]=\sum_{t=0}^{\infty} \frac{\left[\left(a_{p}\right)\right]_{t}(-x)^{t} q^{t(t-1) / 2}}{[1]_{t}\left[\left(b_{l}\right)\right]_{t}[\gamma+t]_{t}} \\
& \times{ }_{p} \Phi_{l+1}\left[\begin{array}{c}
t+\left(a_{p}\right) ; x \\
2 t+\gamma+1, t+\left(b_{l}\right)
\end{array}\right] \cdot{ }_{l+r+2} \Phi_{p+m}\left[\begin{array}{c}
\left(b_{l}\right),\left(c_{r}\right), \gamma+t,-t ; q w \\
\left(a_{p}\right),\left(d_{m}\right)
\end{array}\right] .
\end{aligned}
$$

Then using a technique similar to one used in the deduction of (2.1), we get the general expansion

$$
\begin{array}{r}
{ }_{+t} \Phi_{m+u}\left[\begin{array}{c}
\left.\left(c_{r}\right),\left(e_{t}\right) ; w x\right] \\
\left(d_{m}\right),\left(f_{u}\right)
\end{array}\right]=\sum_{n=0}^{\infty} \frac{\left[\left(a_{p}\right)\right]_{n}\left[\left(e_{t}\right)\right]_{n}(-x)^{n} q^{n(n-1) / 2}}{[1]_{n}\left[\left(b_{l}\right)\right]_{n}\left[\left(f_{u}\right)\right]_{n}[\gamma+n]_{n}} \\
\times{ }_{p+t} \Phi_{l+u+1}\left[\begin{array}{c}
\left(a_{p}\right)+n,\left(e_{t}\right)+n ; x \\
\left(b_{l}\right)+n,\left(f_{u}\right)+n, 2 n+\gamma+1
\end{array}\right] \\
\cdot{ }_{l+r+2} \Phi_{p+m}\left[\begin{array}{c}
\left(b_{l}\right),\left(c_{r}\right), \gamma+n,-n ; q w \\
\left(a_{p}\right),\left(d_{m}\right)
\end{array}\right] .
\end{array}
$$

(3.1) is the $q$-analogue of a result due to Fields and Wimp [7;2.4]. For $p=2, l=0$ and $a_{1}=\alpha, a_{2}=\beta$ it yields (2.1). 
4. In this section, I proceed to deduce a generalisation of the $q$-analogue of Euler's transformation for $a_{2} \Phi_{1}$ viz.,

$$
{ }_{2} \Phi_{1}\left[\begin{array}{c}
a, b ; x \\
c
\end{array}\right]=\left(1-x q^{a+b-c}\right)_{c_{-a-b} \Phi_{1}}\left[\begin{array}{c}
c-a, c-b ; x q^{a+b-c} \\
c
\end{array}\right] .
$$

The result to be proved is:

$$
\begin{aligned}
{ }_{p+1} \Phi_{p}\left[\begin{array}{c}
\left(\alpha_{p+1}\right) ; z \\
\left(\rho_{p}\right)
\end{array}\right] & =\left(1-z q^{s_{p+1}-\sigma_{p}}\right)_{\sigma_{p}-s_{p+1}} \\
& \prod_{r=1}^{p-1} \sum_{n_{r}=0}^{\infty} A_{n_{r}} z^{n_{r}}{ }_{2} \Phi_{1}\left[\begin{array}{c}
\sigma_{p}-S_{p+1}+\gamma_{p-1}, \rho_{p}-\alpha_{p+1} ; z q^{s_{p+1}-\sigma_{p}} \\
\rho_{p}+\gamma_{p-1}
\end{array}\right],
\end{aligned}
$$

where

$$
A_{n_{r}}=\frac{\left[\sigma_{r}-S_{r}+\gamma_{r-1}\right]_{n_{r}}\left[\rho_{r}-\alpha_{r+1}\right]_{n_{r}} \prod_{t=r}^{p-1}\left[\alpha_{t+2}+\gamma_{r-1}\right]_{n_{r}} q^{n_{r}\left(s_{r+1}-\sigma_{r}\right)}}{[1]_{n_{r}} \prod_{t=r}^{p}\left[\rho_{t}+\gamma_{r-1}\right]_{n_{r}}}
$$

and

$$
\begin{array}{r}
S_{r}=\alpha_{1}+\alpha_{2}+\cdots+\alpha_{r} ; \quad \sigma_{r}=\rho_{1}+\rho_{2}+\cdots+\rho_{r}, \quad \gamma_{r}=n_{1}+n_{2}+\cdots+n_{r}, \\
r=1,2,3, \cdots ; \gamma_{0}=1 \text { and }|z|<1,|q|<1 .
\end{array}
$$

For $p=1$, this gives (4.1). To prove the formula (4.2), we multiply both sides by $t^{\alpha_{p+2^{-1}}}(1-q t)_{p_{p+1}-\alpha_{p+2^{-1}}}$ replace $z$ by $z t$ and take the basic integral from 0 to 1 . Then integrating both sides by making use of a result of Jackson [6], we get

$$
\begin{aligned}
& B_{q}\left(\alpha_{p+2} ; \rho_{p+1}-\alpha_{p+2}\right) \Phi\left[\begin{array}{c}
\left(\alpha_{p+2}\right) ; z \\
\left(\rho_{p+1}\right)
\end{array}\right]=\prod_{r=1}^{p-1} \sum_{n_{r}=0}^{\infty} A_{n_{r}} z^{n_{r}} \\
& \quad \times \sum_{n_{p}=0}^{\infty} \frac{\left[\sigma_{p}-S_{p}+\gamma_{p-1}\right]_{n_{p}}\left[\rho_{p}-\alpha_{p+1}\right]_{n_{p}} z^{n_{p}} q^{n_{p}\left(s_{p+1}-\sigma_{p}\right)}}{[1]_{n_{p}}\left[\rho_{p}+\gamma_{p-1}\right]_{n_{p}}} \\
& \quad \times S_{0}{ }^{1} t^{\gamma_{p}+\alpha_{p+2}-1}(1-q t)_{\rho_{p+1}-\alpha_{p+2}-1}\left(1-z t q^{S_{p+1}-\sigma_{p}}\right)_{\sigma_{p}-s_{p+1}} d(q, t) \\
& =\prod_{r=1}^{p-1} \sum_{n_{r}=0}^{\infty} A_{n_{r}} z^{n_{r}} \sum_{n_{p}=0}^{\infty} \frac{\left[\sigma_{p}-S_{p}+\gamma_{p-1}\right]_{n_{p}}\left[\rho_{p}-\alpha_{p+1}\right]_{n_{p}}}{[1]_{n_{p}}\left[\rho_{p}+\gamma_{p-1}\right]_{n_{p}}} \\
& \quad \times B_{q}\left[\gamma_{p}+\alpha_{p+2} ; \rho_{p+1}-\alpha_{p+2}\right] z^{n_{p}} q^{n_{p}\left(S_{p+1}-\sigma_{p}\right)} \\
& \quad \times{ }_{2} \Phi_{1}\left[\begin{array}{c}
S_{p+1}-\sigma_{p}, \gamma_{p}+\alpha_{p+2} ; z \\
\rho_{p+1}+\gamma_{p}
\end{array}\right] .
\end{aligned}
$$

Then applying (4.1) on the right-hand side of (4.3), and dividing throughout by $B_{q}\left[\alpha_{p+2} ; \rho_{p+1}-\alpha_{p+2}\right]$, we get the formula (4.1) with $(p+1)$ in place of $p$, which completes the induction proof. This is the basic analogue of a result due to MacRobert $[2 ; 29$, p. 363$]$.

5. In this section, I deduce the basic generalisation of Saalschütz's theorem. To do so, multiply both sides of $(4.1)$ by $(1-z)_{s_{p+1}}-\sigma_{p}$ and equating the coefficient 
of $z^{n}$ on both the sides and simplifying, we get the required result in the form:

$$
\begin{gathered}
\Phi\left[\begin{array}{c}
\left(\alpha_{p+1}\right),-n ; q \\
\left(\rho_{p}\right), 1-\sigma_{p}+S_{p+1}-n
\end{array}\right]= \\
\times \prod_{r=1}^{p-1} \sum_{n_{r}=0}^{n-\gamma_{r}-1} \frac{\left[\sigma_{p}-S_{p}\right]_{n}\left[\rho_{p}-\alpha_{p+1}\right]_{n}}{\left[1 \sigma_{r}\right]_{n_{r}}\left[\sigma_{p}-S_{r}+S_{p}+\gamma_{r-1}\right]_{n_{r}}\left[\rho_{r}-\alpha_{r+1}\right]_{n_{r}}\left[S_{n_{r}}\left[\gamma_{r-1}-n\right]_{n_{r}}\right.} \\
\times \frac{\prod_{t=r+2}^{p+1}\left[\alpha_{t}+\alpha_{p+1}+\gamma_{r-1}\right]_{n_{r}}}{\prod_{t=r}^{p-1}\left[\rho_{t}+\gamma_{r-1}\right]_{n_{r}}} q^{n_{r}\left(S_{r+1}-\sigma_{r}\right)+\gamma_{p-1}\left(\sigma_{p-1}-S_{p}+1\right)} .
\end{gathered}
$$

This reduces to the basic analogue of the Saalschütz theorem $[1 ; 8.4(1)]$ for $p=1$. Moreover (5.1) is also the basic analogue of a result due to MacRobert $[2 ; 30$, p. 365]:

In particular, taking $p=3$ and $\alpha_{1}=a, \alpha_{2}=b, \alpha_{3}=c, \alpha_{4}=d ; \rho_{1}=e, \rho_{2}=f$; $\rho_{3}=g$ in (5.1) we get the basic analogue of another result due to MacRobert [2: p. 366], viz.,

$$
\begin{aligned}
& { }_{5} \Phi_{4}\left[\begin{array}{c}
a, b, c, d,-n ; q \\
e, f, g, 1+a+b+c+d-e-f-g-n
\end{array}\right] \\
& =\frac{[e+f+g-a-b-c]_{n}[g-d]_{n}}{[e+f+g-a-b-c-d]_{n}[g]_{n}} \\
& \times \sum_{p=0}^{n} \frac{[e-a]_{p}[e-b]_{p}[-n]_{p}[c]_{p}[d]_{p} q^{p(f-c+1)}}{[1]_{p}[e+f+g-a-b-c]_{p}[1+d-g-n]_{p}[e]_{p}[f]_{p}} \\
& \times{ }_{4} \Phi_{3}\left[\begin{array}{c}
e+f-a-b+p, f-c, p-n, p+d ; q \\
e+f+g-a-b-c+p, 1-g+d+p-n, f+p
\end{array}\right] .
\end{aligned}
$$

Further, putting $g=c$ and writing $c$ for $d$ and $f$ for $g$ in (5.2), we have

$$
\begin{aligned}
{ }_{4} \Phi_{3}\left[\begin{array}{c}
a, b, c,-n ; q \\
e, f, 1+a+b+c-e-f-n
\end{array}\right]=\frac{[e+f-a-b]_{n}[f-c]_{n}}{[e+f-a-b-c]_{n}[f]_{n}} \\
\times{ }_{4} \Phi_{3}\left[\begin{array}{c}
c, e-b, e-a,-n ; q \\
e+f-a-b, e, 1+c-f-n
\end{array}\right],
\end{aligned}
$$

which is a basic analogue of a result due to Whipple [10]. Further applying (5.3) to the right-hand side of (5.3) we get the basic analogue of still another result due to Whipple [10].

My thanks are due to Dr. R. P. Agarwal for his help during the preparation of this paper. I am also thankful to the Council of Scientific and Industrial Research for awarding me a Junior Research Fellowship.

The University,

Lucknow, India

1. W. N. BAILEx, Cambridge Tract on Generalised Hypergeometric Series, Cambridge, 1935.

2. T. M. MACRobert, Functions of a Complex Variable, V-edition, London, 1962. 
3. R. P. Agarwal, "Some hypergeometric identities," Ann. Soc. Sci. Bruxelles Sér. I, v. 68,1953 , pp. 186-202. MR 15, 421 .

4. W. HAнN, "Úber die höheren Heineschen Reihen und eine einheitliche Theorie der sogenannten speziellen Functionen," Math. Nachr., v. 3, 1950, pp. 257-294. MR 12, 711.

5. W. HAHN, "Beiträge zur Theorie der Heineschen Reihen," Math. Nachr., v. 2, 1949, pp. 340-379. MR 11, 720 .

6. F. H. JACKSON, “q-difference equations," Amer. J. Math., v. 32, 1910, pp. 305-314.

7. J. L. FinLd \& J. WIMP, "Expansion of hypergeometric functions in hypergeometric functions," Math. Comp., v. 15, 1961, pp. 390-395.

8. L. Toscano, "Teorema di moltiplicazione sulle funzioni ipergeometriche generalizzate," Ann. Mat. Pura Appl., (4), v. 44, 1956, pp. 269-288. MR 17, 1206.

9. Y. L. LUKE \& R. L. Coleman, "Expansion of hypergeometric functions in series of other hypergeometric functions," Math. Comp., v. 15, 1961, pp. 233-238.

10. F. J. W. WHIPpLe, "Some transformations of generalised hypergeometric series," Proc. London Math. Soc., v. 22, 1927, pp. 257-272.

\section{Boundedness of Difference Kernels of Bessel and Fourier Series}

\section{By Shih-Hsiung Tung}

1. Introduction. Let $\alpha_{m}, m=1,2, \cdots$, be positive zeros of Bessel functions $J_{\nu}(x)$ of the first kind of order $\nu \geqq-\frac{1}{2}$, arranged in increasing order. The kernels of Bessel and Fourier cosine series on $[0,1]$ are denoted as

$$
B_{M}(x ; t)=\sum_{m=1}^{M} 2 t J_{\nu}\left(\alpha_{m} x\right) J_{\nu}\left(\alpha_{m} t\right) J_{\nu+1}^{2}\left(\alpha_{m}\right)
$$

and

$$
C_{M} \equiv C_{M}(x ; t)=1+\sum_{m=1}^{M} 2 \cos (m \pi x) \cos (m \pi t)
$$

We define the difference kernel to be

$$
D_{M}(x ; t)=B_{M}(x ; t)-C_{M}(x ; t) .
$$

Two series $S=\sum s_{i}$ and $T=\sum t_{i}$ are said to be equiconvergent if $\lim _{n \rightarrow \infty}\left(S_{n}-T_{n}\right)=0$, where $S_{n}$ and $T_{n}$ are partial sums of the first $n$ terms of the series.

Here we study the boundedness (Theorems 1 and 2) of the difference kernel and the equiconvergence (Theorem 3) of Bessel series of a Lebesgue integrable function on $[0,1]$ and its corresponding Fourier cosine series. The proof of the boundedness of the difference kernel of two series is mainly based on the application of the asymptotic expansion of Bessel functions and their zeros. The equiconvergence theorem, which is a direct application of Theorem 2, is a stronger result obtained by a simple and straightforward proof comparable to the analogous ones given in [6] and [8]. We notice that the cosine series may equally well be replaced by a sine or sine and cosine series.

2. Preliminaries. The following results are needed later.

LeмmA 1. If $a$ is real, $b \geqq 0$ and $0<\eta \leqq y-k \leqq 1-\eta<1$ for some integer

Received May 20, 1965 